\section{Prädiktoren für einen vorzeitigen Ausstieg aus der Hormontherapie}

\begin{abstract}
Adjuvante Hormontherapien auf der Basis von Tamoxifen oder Aromatasehemmern vermindern deutlich das Rezidivrisiko und die krankheitsbezogene Mortalität beim Mammakarzinom. Vorzeitige Therapieabbrüche, häufig wegen behandlungsbezogener Nebenwirkungen, konterkarieren diesen Effekt.
\end{abstract}

$\mathrm{D}$ urch die vorliegende Studie mit Daten aus mehreren schwedischen Registern und einer großen, bevölkerungsbasierten Kohorte sollten Vorhersagevariablen für den vorzeitigen Abbruch einer adjuvanten Hormontherapie des Mammakarzinoms identifiziert werden, darunter Baseline-Prädiktoren wie Hormonersatztherapien, Lebensstilfaktoren, sozioökonomischer Status, familiäre Vorbelastung durch Mamma- oder Ovarialkarzinom und frühe therapieassoziierte Faktoren. Die Forscher identifizierten 3.470 Patientinnen, die zwischen 2005 und 2008 die Diagnose Brustkrebs erhalten und eine adjuvante Hormontherapie mit Tamoxifen oder Aromatasehemmern begonnen hatten.

Als Baseline-Prädiktoren für den vorzeitigen Abbruch einer adjuvanten Hormontherapie wurden die Einnahme von Analgetika, Sedativa, Antidepressiva oder Medikamenten zur Behandlung gastrointestinaler Beschwerden ein Jahr vor der Brustkrebsdiagnose identifiziert. Die Einnahme von Hormonersatzpräparaten ein Jahr vor der Diagnose korrelierte mit einer höheren Abbruchrate nur im ersten Jahr. Weitere BaselinePrädiktoren waren jüngeres ( $<40$ Jahre) oder höheres ( $\geq 65$ Jahre) Alter zum Diagnosezeitpunkt, ein erhöhter CharlsonKomorbiditätsindex und ein Ovarialkarzinom in der Familienanamnese. Der Gebrauch symptomlindernder Medikamente im ersten Behandlungsjahr erwies sich als Prädiktor für den Abbruch in den 4 Folgejahren. Dabei korrelierte die Abbruchrate mit der Anzahl eingenommener Medikamente. Das Abbruchrisiko erhöhte sich um 50 \% für Patientinnen, wenn die Therapie einen Wechsel von Tamoxifen auf Aromatasehemmer oder vice versa vorsah.

Fazit: Diese Variablen können als Basis für zielgerichtete Interventionen dienen, um vorzeitige Abbrüche einer adjuvanten Hormontherapie zu verhindern und damit die Prognose der Patientinnen zu verbessern.

Wolfgang Zimmermann

He W et al. Predictors of discontinuation of adjuvant hormone therapy in patients with breast cancer. J Clin Oncol. 2015;33(20):2262-9.

\section{Patientenbezogene Resultate der Hormontherapie des frühen Mammakarzinoms}

\author{
Die kombinierte Wirksamkeitsanalyse der Studien TEXT und SOFT zeigte \\ einen signifikanten Vorteil hinsichtlich des krankheitsfreien Überlebens (DFS) \\ für Exemestan plus ovarielle Suppression im Vergleich zu Tamoxifen plus \\ ovarielle Suppression. Wird dieser Vorteil mit mehr endokrinen Symptomen \\ und einer schlechteren Lebensqualität (QoL) erkauft?
}

\footnotetext{
Z wischen 2003 und 2011 erhielten 4.717 prämenopausale Frauen mit hormonrezeptorpositivem Brustkrebs in den Studien TEXT oder SOFT unverblindet über 5 Jahre eine adjuvante Therapie mit Exemestan plus OFS („ovarian function suppression“) oder Tamoxifen plus OFS. Zur OFS standen drei Alternativen zur Verfügung: 1. Triptorelin, ein GnRH(„gonadotropin-releasing hormone")-Analogon, 2. die bilaterale Oophorektomie oder 3. Bestrahlung. Eine zusätzliche Chemotherapie war möglich. Zur Erfassung der patientenbezogenen Resultate (PRO, „patient reported outcome") füllten die Patientinnen zu Beginn, sowie in den Jahren $1+2$ alle 6 Monate und in den Jahren 3-6 alle 12
}

Monate den QoL-Fragebogen der Interfortgeführt.
Vagina, eine stärkere Abnahme des sexuellen Interesses und Schwierigkeiten, erregt $\mathrm{zu}$ werden; diese Unterschiede blieben über die Zeit bestehen. Unter Exemestan/OFS war die Zunahme von Knochen- oder Gelenkschmerzen, vor allem zum 6-Monats-Assessment, stärker ausgeprägt als bei den Patientinnen unter Tamoxifen/OFS. Die Veränderungen der globalen QoL-Domänen gegenüber Baseline waren gering und zwischen den beiden Behandlungsarmen während der 5 Jahre vergleichbar. national Breast Cancer Study Group aus. Die Intention-to-treat-Auswertung erfolgte nach einem medianen Follow-up von 5,7 Jahren (Interquartilen-Range [IQR] 3,7-6,9); Therapie und Nachbeobachtung der Patientinnen werden weiter

In den ersten 5 Jahren litten die Patientinnen (median 43 Jahre alt) unter Tamoxifen/OFS stärker unter Flush und Schweißausbrüchen als diejenigen unter Exemestan/OFS, obwohl sich diese Symptome mit der Zeit besserten. Auf der anderen Seite berichteten die Frauen in der Exemestan/OFS-Gruppe im Vergleich zu Frauen der Tamoxifen/OFSGruppe verstärkt über Trockenheit der
Fazit: Insgesamt gibt es im Hinblick auf die Lebensqualität kein starkes Argument für eine der beiden Kombinationen. Die Entscheidung für eine der beiden Therapien, Tamoxifen/OFS oder Exemestan/OFS, sollte abhängig von der Belastung der jeweiligen Patientin durch die endokrinen Symptome individuell erfolgen.

Brigitte Schalhorn with adjuvant exemestane versus tamoxifen in premenopausal women with early breast cancer undergoing ovarian suppression (TEXT and SOFT): a combined analysis of two phase 3 randomised trials. Lancet Oncol. 2015;16(7):848-58.
Bernhard J et al. Patient-reported outcomes 